\title{
Note on terminology
}

In theory, cryptology is a discipline composed of two fields, cryptography, that is secret writing, and cryptanalysis, that is codebreaking (cryptanalysis is a modern term forged by William Friedman). In the period under study, no such methodical distinction was used, ciphering, encryption, "translating", "working with chiffres" and many other terms are applied somewhat inconclusively in the sources. Therefore, throughout the book, differentiation between cryptology and cryptography will be neither systematic nor analytic. Whenever I refer to the practice of ciphering in general, I will use cryptography, unless I want to particularly emphasize that besides encryption, codebreaking is also included in the activity, because then I will use cryptology.

All other terms - open text, plain text, monoalphabetic, homophonic, and polyalphabetic ciphers, frequency analysis, probable word method, entropy, etc. - will be explained in the book at their first occurrences. 
